\title{
The design and Implementation of Sports Dance Teaching System Based on Digital Media Technology
}

\author{
Guiyou Liu \\ \{ liuguiyoucq@126.com\}
}

Basic Course Department, Chongqing College of Finance and Economics Chongqing 402160, China

\begin{abstract}
In this paper, the application of virtual reality simulation technology of sports dance rehearsals were preliminary research and implementation of a virtual simulation system of sports dance rehearsal and rehearsal, the system can for the coordination of the sports dance creating staff on formation of the arrangement, the movements of the mechanical rationality and sound dance provide technical parameters, can be used as a powerful auxiliary tool for composing personnel of sports dance. The main research contents of this paper include: first to based on the physical simulation research $111 \mathrm{e}$ status has made the review and the summary, in order to carry out computer simulation of sports dance performance. This paper is divided into three blocks of dance in the process can not be avoided in the virtual human animation technology, dancers and edit dance of harmony harmony is discussed. At last, the three pieces of work are integrated into the final system of sports dance choreography. In the virtual human animation technology, the traditional way for the teacher to carry out a frame by frame manual labeling, heavy workload, high cost of motion capture, manual identification accuracy. In this paper, we use a combination of linear interpolation and quaternion interpolation method to capture the motion information of interpolation, to solve the problem of hopping between some separate interpolation algorithm for every frame of the animation, so that eventually the synthesis of human animation is natural, smooth and realistic. Initial animation still has a lot of problems, in order to allow the computer to make action of modified return to action subject, this paper of each rigid body motion clips do accurate and detailed data analysis, use based on keyframe the spline interpolation algorithm to solve location bias, the quaternion in value algorithm to solve the action subject or body movements rotation problem, let the computer auxiliary action scheduling system make actions to modify the fully return to virtual human animation.
\end{abstract}

Keywords: Virtual environment; sports dance; virtual crowd simulation; collision detection; path planning.

\section{Introduction}

Digital media technology computer is through the combination of a variety of text, graphics, image, sound, animation and video media, to them for sampling and quantization, editing, coding and compression, reconstruction display and storage, transmission and processing, and to establish a logical connection. With its incomparable advantages such as convenient, accurate, efficient, convenient storage and easy to modify, the computer constantly refresh the record. In recent years the rapid development of multimedia technology, software upgrade, let the computer is widely used in the field of design performance won the 
broad prospects, multimedia technology not only to pay new forms to the stage of visual performance brought a revolution, people's aesthetic philosophy also along with hair gave birth to great changes. With the wide application of multimedia technology, choreography is breaking through the limitations of traditional manual era and closed, stage is designed into an art of time and space [1], the combination of complex, both of which music literature, with time, aural, painting, building the function of the space, visual choreography not only to expand the visual range of the viewer and editor, expand people's thinking ability. Computer technology in dance creation and graphic design plays an important role, sports dance practitioners to provide the new train of thought, the computer can become a new "stage language", people have enough reason to believe combined with computer and sports dance will stimulate more creative passion and artistic spirit. Virtual reality technology is characterized by low cost, high simulation decision has broad application prospects in the field of physical simulation. Virtual reality technology can not only for the sports dance practitioners and athletes to provide new salary, effective training methods, and expand the scope of application of the simulation system. This, in turn, could promote the development of virtual reality technology. The so-called VR based sports simulation, is the use of virtual reality technology to simulate the process of physical training so that teachers and students can be in a virtual environment for training.

\section{Auxiliary System Structure of Sports Dance Movement Arrangement}

How in the film, animation, games, the performance of a realistic physical action is directly related to the quality of the final product, so the motion synthesis technology in the modern animation film and television industry has received more and more attention. The traditional method has the very high request to the personal quality of the practitioner, the workload is very big. In order to facilitate the rapid synthesis of lifelike body movements, researchers have proposed a lot of new methods, greatly reducing the workload of the relevant personnel, improve the quality of the product. The picture shows the Microsoft and the Kineet tracking human posture and movement.

Body technology movement synthesis technology in general can be divided into three categories:

Artificial method. This approach mainly rely on manual to operate the binary code is complete, time-consuming and precision is not high, the final result of the synthesis is not good.

Device based method. This method is much more than the first method in data acquisition and sorting, but it is only suitable for a small number of experts, especially for the relevant hardware, so the method is difficult to be popularized [2].

Method for synthesizing action with data and virtual human. The method of comprehensive computer technology and people combine the efficiency and effectiveness of the first two methods are improved a lot. 


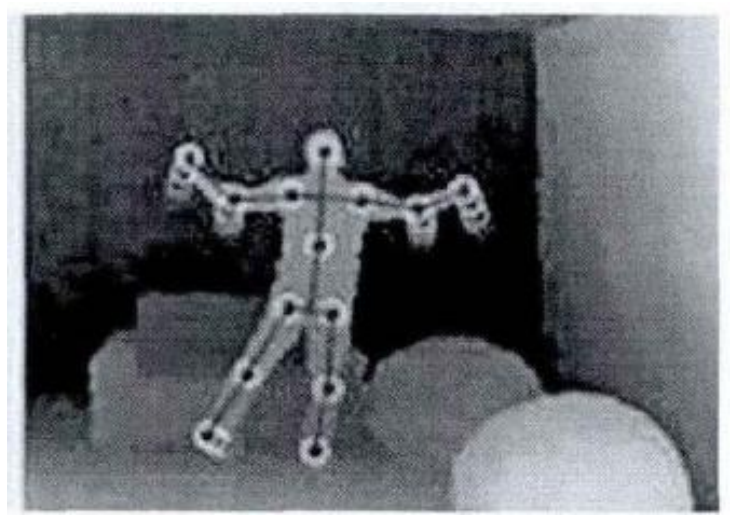

Fig. 1. Use the robot to track the body movements

\subsection{Sports Dance Movement Arrangement Auxiliary System}

In the data layer of the connection action library selected can be smoothly connected to the difficulty action of the connection action". Main consideration between virtual human collision detection and path to avoid for path using the tool set layer "motion path editing module dance designers can further new motion path specified by the movement, the stage of re editing. After the completion of the above work, dance designers can be to design good sports preview for further modification, the choreographer of the corresponding to each passages of motion design after the completion of the can save the motion, and design corresponding to the next passages motion or out of the system. In order to ensure a smooth transition between the design of multi segment motion, the system will automatically synthesis of motion transition between adjacent passages corresponding to the motion clip. These specified "connection action" as "candidate connection action" feedback to the dance designer, dance designer in the candidate connection action among the selection to join the connection action. Reuse tool layer "motion connection to connect the motion segment, this time of movement as a whole will appear very raw sugar," motion blending will slip motion smoother, finally, the "sports equipment synthesis" join the instrument and three modules combined order can dance to designer's requirement of smooth continuous motion clips are satisfied [3].

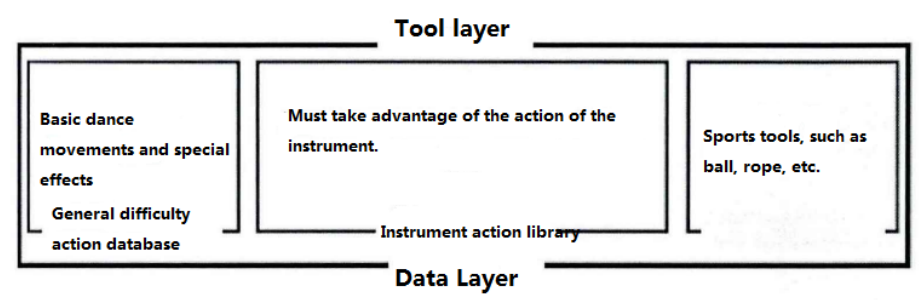

Fig. 2. The overall structure of the auxiliary system of sports dance choreography

\subsection{Motion Database Design}

The use of motion sensing device can get accurate athletes, angular motion, but these movements only specific people to act in a particular manner, specific movement, strong 
personal color makes computer workers is difficult to motion data to modify reuse. Therefore, the authors add additional information about the movement of the library in the design of the database, which mainly include inter movement constraints, inter movement relation and classification. The motion graph of several vertices can be obtained from dance designer to specify a number of difficult movements, starting from the vertices can be connected vertex edge ", these" edge "is actually used to action for connecting" difficulty ", due to connect two vertices of the edge may be more than a, then multiple connection movements can as connection action" candidate "to dance in the designer. Is listed in this paper, based on university sports dance course required manual complete sets of actions selected out of twenty "difficulty", peripheral rectangular box represents the movement, nine "action" sample connection, elliptical box represents is connected with the motor. The connection motion of the ellipse frame can be connected with any two difficult actions to obtain a new motion segment [4].

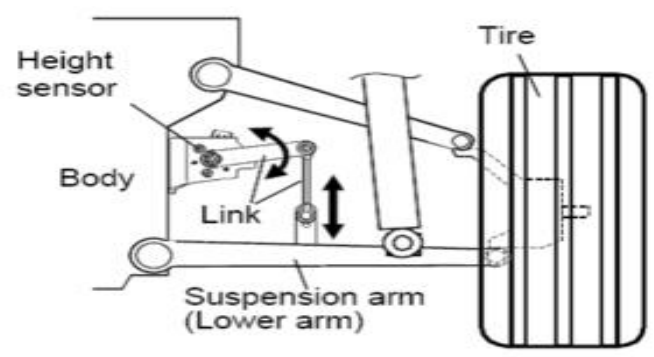

Fig. 3. Schematic diagram of motion sensor structure

\section{Motion Editing}

Virtual human motion generation and interactive control of virtual human is a hot and difficult research topic in the field of virtual simulation. Human motion sensor can be used to obtain the human body motion data, but the virtual human body driven by the captured data is a process of real motion representation, which is difficult to be reused. Motion editing algorithm is based on the human motion tracking technology to generate a realistic, image and meet the needs of users of the new motion sequence. We not only require the synthesis of human motion is natural and realistic, but also want to be able to provide user programming interface in order to human-computer interaction, according to the user's requirements to adjust the corresponding action. The generation of the new action sequence not only makes the user can control the high level, but also makes the motion capture data reuse. It can be widely used in the field of animation, games, virtual reality and so on. The formation of the new motion sequence includes three steps: the target action requirements, including the type of the target action, length and other basic information. Arrange movement, according to the requirements of the use of motion capture to the movement of the fragments of the synthesis of new motion clips. Smooth transition, the final synthesis of the animation does not meet the requirements of mechanics or the impact of modification.

How to ensure the smooth flow of the virtual motion sequence is the target of this paper. In this paper, we do not modify the real motion data captured, but make use of the three 
methods of motion image, affine transformation and offset mapping to splice the two segment motion segment. Mirror movements in the replication source action "and" the target action "played a key role, affine transformation helps animation designers removed from the size differences between the source and target objects introduced error, displacement mapping of single direction movement calculation the effective correction. Author in the repeated simulation of traditional algorithms in the process found tail current a motion segment inclination and after an action the first meal difference is large, even though the interpolation calculate intermediate meal can not guarantee animation synthesis complete, smooth and real, and will introduce a large amount of computation. In the actual project, it is possible to agree that the tail frame of the previous action segment and the first meal of the latter act are similar, which is consistent with the real life. Based on this agreement, the algorithm of the algorithm to the animation of the interpolation algorithm to absorb the three kinds of mature tools: "motion image", "affine transformation", "offset mapping" [5].

\section{Conclusion}

In the virtual human animation technology, the traditional way for the dance designers to carry out a frame by frame manual marking, a lot of work, high cost of motion capture, manual identification accuracy. Based on linear interpolation and quaternion interpolation two interpolation method for interpolating in the capture of real human motion information, both to ensure the animation synthesis of smooth and realistic, and avoids the simple use of jump caused by linear interpolation of the animation. In order to make the modified results redirect to the top of the virtual human, for each action were accurate data analysis, and through the action of virtual human make the results in the 3D scene reproduce, the splines for the position shift is not sensitive to the key frame interpolation method, and for the angles of rotation of the changes we use quaternion interpolation method. Simulation results show that students of each movement can be real-time, multi angle display, realizes from experience based training analysis method to human motion modeling and Simulation of human motion analysis method based on the transformation.

\section{References}

[1] Tang Wei, Rao Yan. Design of digital fantasy in the sports dance art research and application. Journal of Wuhan Institute of physical education. No. 10. (2014)

[2] Peng Jianxiang. The marriage of. Art and multimedia choreography. No. 08. (2009)

[3] Li Yan, Dong Fei. Design of the simulation system of Physical Education (martial arts). Journal of Shandong Sports Institute. No. 09. (2012).

[4] Sun Shouqian, Wang Xin, Diao Zaizhen, Chun Yan Deng. Artistic gymnastics auxiliary arrangement system. Journal of Computer Aided Design \& computer graphics Journal of. No. 02. (2015)

[5] Gao Jianxin, Yang Hongxia, Yang Hongmei, Cui Liang. The theory of the four elements and the application of the virtual reality technology. Journal of Hebei Polytechnic University (Natural Science Edition). No. 03. (2012) 